\title{
EFFECT OF A PESTICIDE, PENTACHLOROPHENOL (PCP) ON SOIL MICROFLORA \\ II. EFFECT OF PCP ON BACTERIAL FLORA IN SOIL PERCOLATED WITH GLYCINE OR WATER
}

\author{
KYO SATO \\ Institute for Agricultural Research, Tohoku University, \\ 2-1-1, Katahira, Sendai 980, Japan
}

(Received January 14, 1985)

\begin{abstract}
The soil bacterial floras were studied in glycine- and water-percolated soils with or without PCP treatment. Many bacterial strains isolated from the percolated soils with PCP treatment died after purification, while those from the percolated soils without PCP did not die. Of the living strains, the bacterial flora in water-percolated soil with PCP treatment was composed of one specific bacterial group: gram-negative, coccoidal rod-shaped, polarly flagellated, Kovacs' oxidase positive, and neither oxidative nor fermentative in Hugh and Leifson's test. On the other hand, the bacterial flora in water-percolated soil without PCP was composed of several bacterial groups. In the glycine-percolated soil without PCP treatment one group of bacteria predominated: gramnegative, rod-shaped, polarly flagellated, Kovacs' oxidase positive, oxidative acid production from glucose in Hugh and Leifson's test, gelatin-liquefaction negative, nitrate-reduction positive, and alkaline reaction to $\mathrm{BCP}$ milk. However, different groups of bacteria were isolated from the glycine-percolated soil treated with PCP. Almost all of the strains isolated from the glycine-percolated soil without PCP treatment readily utilized glycine for their growth, but those isolated from the soil with PCP added utilized glycine scantily for their growth. These results indicate that PCP affects soil bacterial flora and that the bacteria responsible for glycine degradation in the glycine-percolated soil treated with PCP are different from the bacterial flora in soil without PCP, even though the apparent patterns of both glycine-degradation and the increase of bacterial population did not change in the presence of PCP.
\end{abstract}

Many reports on the relation between pesticides and soil microorganisms are focussed on the degradation of pesticides in unsterilized soils $(1,2)$, or by micro- 
organisms isolated from soil $(3,4)$. Studies on the effect of pesticides on soil microorganisms have been carried out from the view-point of soil biochemistry by measuring microbial activities such as $\mathrm{CO}_{2}$ evolution, ammonification, nitrification, etc. (5-8). Some research has been reported on the quantitative microbial changes in soil at one time after the addition of pesticides $(9,10)$, but there are not many studies on successive quantitative microbial changes (11-13). Of qualitative microbial changes, the changes in the populations of two or three kinds of microbial groups such as bacteria, actinomycetes, and fungi have been followed $(9,13)$, but follow-up surveys of one kind of microbial group at the generic level are very scarce (14).

In a previous paper (15) I reported that the same pattern of glycine-degradation, accompanied by an increase in numbers of bacteria, occurred in glycinepercolated soil whether PCP was added or not. This seems to indicate that PCP does not affect either bacteria or the degradation of glycine in the soil.

The present report, therefore, aims at the confirmation of the effect of PCP on bacterial flora in the percolated soils. For this purpose, the bacterial strains isolated from several plots of the percolated soil were assorted taxonomically and also tested for their nutritional characteristics.

\section{MATERIALS AND METHODS}

Bacterial strains. The 65 strains studied were isolated from percolated soils (15). Isolation was performed randomly from colonies on agar plate medium which had been inoculated with soil dilutions of each of the percolated soils in order to count the number of bacteria in the percolated soils. Fifteen strains were isolated from the water-percolated soils with and without PCP treatment respectively on the 5th day of percolation when the population of bacteria reached the maximum number in soils. The strains which were isolated from the waterpercolated soil without PCP added were termed W-strains, and those from the soil with PCP treatment P-strains. Thirty strains were isolated from the glycinepercolated soil without PCP added on the 5th day of percolation and were termed G-strains. Thirty strains were also isolated from the glycine-percolated soil with PCP treatment on the 5th and 15 th days of percolation, because the bacterial population that attained the maximum number was maintained for a long time during percolation in the percolated soil. The strains isolated on the 5th and 15th days were termed GP5-strains and GP15-strains, respectively. Of a total of 120 strains isolated, 55 strains died while the cultures were stored.

The composition of the isolation medium (albumin agar medium) was as follows (wt/vol percentages): egg albumin, $0.025 \%$; glucose, $0.1 \% ; \mathrm{K}_{2} \mathrm{HPO}_{4}$, $0.05 \% ; \mathrm{MgSO}_{4} \cdot 7 \mathrm{H}_{2} \mathrm{O}, 0.02 \% ; \mathrm{Fe}_{2}\left(\mathrm{SO}_{4}\right)_{3}$, trace; yeast extract, $0.005 \%$; agar, $1.3 \%$. pH was adjusted to 7.2 with $1 \mathrm{~N} \mathrm{NaOH}$. The isolates were grown and maintained on slants of the albumin agar medium. 
Phenotypic tests. A total of 18 characteristics were determined for each strain. Incubation was at $25^{\circ} \mathrm{C}$ during the respective tests.

Gram staining was done by the usual method (16): phenolic gentian violet staining followed by counter-staining with safranin. Motility was determined from wet mounts, and flagellum arrangement assessed with an electron microscope. Cultural characteristics were examined for the production of pigment by the colony on the albumin agar medium after 7 days of incubation. Production of water soluble greenish yellow pigment was also examined in liquid albumin medium (without agar) supplemented with $0.5 \%$ (wt $/ \mathrm{vol}$ ) glycerol at the final concentration, a modification of a reported method (17). Heat-resistance was determined by the growth of cells after heat-treatment of the thick cell suspension of aged cultures at $80^{\circ} \mathrm{C}$ for $10 \mathrm{~min}$.

Cultures grown for $24 \mathrm{hr}$ on the albumin agar medium were tested for catalase by adding $3 \%$ (wt/vol) $\mathrm{H}_{2} \mathrm{O}_{2}$ and for cytochrome oxidase production using Kovacs' reagent (18). Acid production from D-glucose, lactose or sucrose was detected both aerobically and anaerobically in a basal medium with $0.5 \%$ of the final sugar concentration. The composition of the basal medium was as follows (wt/vol percentages): polypeptone, $0.2 \% ; \mathrm{NaCl}, 0.5 \% ; \mathrm{K}_{2} \mathrm{HPO}_{4}, 0.03 \%$; bromothymolblue, $0.003 \%$; agar, $0.3 \%$. For the nitrate reduction test, a liquid medium was used consisting of (wt/vol percentages) $1.0 \%$ polypeptone, $0.5 \% \mathrm{NaCl}, 0.1 \%$ $\mathrm{KNO}_{3}$, and $1.0 \%$ meat extract. Nitrite was detected with $\alpha$-naphthylaminesulfanilic acid reagent (19), and residual nitrate was detected with zinc dust.

Gelatin liquefaction was tested on the nutrient broth medium containing $1 \%$ (wt/vol) gelatin. Stab culture was performed at $30^{\circ} \mathrm{C}$ for 10 days, and the liquefaction of gelatin was confirmed by cooling the culture in water below $15^{\circ} \mathrm{C}$. Reaction to $\mathrm{BCP}$ milk was tested in a medium composed of (wt/vol percentages) skim milk, 10\%; bromocresolpurple (BCP) as alcohol solution, $0.002 \%(20)$. The cultures were observed daily for 10 days to detect the reaction of $\mathrm{BCP}$ and the peptonization of milk.

Indol production was tested in a medium consisting of (wt/vol percentages): polypeptone, $1.0 \% ; \mathrm{NaCl}, 0.5 \%$ and tryptophan, $0.05 \%$. The presence of indol was indicated with Kovacs' reagent (23). The methyl red and Voges-Proskauer (V.P.) tests were made in a medium consisting of (wt/vol percentages): $0.005 \%$ $\mathrm{H}_{3} \mathrm{PO}_{4}, 0.5 \%$ polypeptone, and $0.2 \%$ glucose (19).

Assimilation of $p$-hydroxybenzoate or glucose was tested by growing the strains in a basal salt solution composed of (wt/vol percentages) $\mathrm{KH}_{2} \mathrm{PO}_{4}, 0.02 \% ; \mathrm{K}_{2} \mathrm{HPO}_{4}$, $0.05 \% ; \mathrm{MgSO}_{4} \cdot 7 \mathrm{H}_{2} \mathrm{O}, 0.02 \% ; \mathrm{NaCl}, 0.02 \% ; \mathrm{CaCl}_{2} \cdot 2 \mathrm{H}_{2} \mathrm{O}, 0.005 \%$ and $\mathrm{FeSO}_{4} \cdot$ $7 \mathrm{H}_{2} \mathrm{O}, 0.0025 \%$. $\quad 0.5 \%$ (wt $\left./ \mathrm{vol}\right) p$-hydroxybenzoate or glucose and $0.2 \%$ (wt $/ \mathrm{vol}$ ) $\mathrm{NH}_{4} \mathrm{NO}_{3}$ were added to the basal salts solution at the final concentrations.

Nutritional tests were done in the basal salts solution supplemented with each substrate at the final concentration of $0.5 \%(\mathrm{wt} / \mathrm{vol})$, and the basal salt solution was also supplemented with $0.005 \%$ (wt $/ \mathrm{vol}$ ) of yeast extract to determine yeast 
Table 1. Phenotypic

\begin{tabular}{|c|c|c|c|c|c|c|c|c|}
\hline $\begin{array}{l}\text { Strain } \\
\text { No. }\end{array}$ & 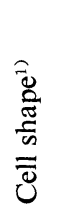 & 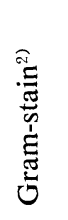 & 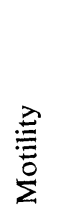 & 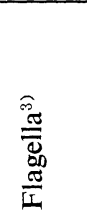 & 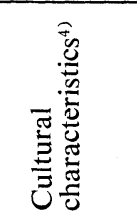 & 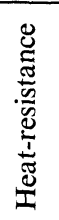 & $\begin{array}{l}\frac{\mathscr{J}}{\tilde{J}} \\
\frac{\tilde{J}}{\tilde{J}}\end{array}$ & 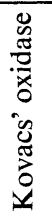 \\
\hline G-3 & $\mathrm{r}$ & - & - & - & yell. w. & - & + & \pm \\
\hline G-15 & sr & - & - & - & yell.w. & - & + & - \\
\hline $\mathrm{W}-1$ & $\mathrm{sr}$ & - & - & - & w. trans. & - & + & - \\
\hline $\mathrm{W}-2$ & $\mathrm{r}$ & - & - & - & br. w. & - & + & - \\
\hline W-9 & $\mathrm{sr}$ & - & - & - & w. & - & + & - \\
\hline $\mathrm{W}-14$ & $\mathrm{cr}$ & - & - & - & w. & - & + & - \\
\hline $\mathrm{W}-15$ & $\mathrm{cr}$ & - & - & - & w. & - & + & - \\
\hline GP15-1 & $\mathrm{sr}$ & - & + & per & w. trans. & - & + & - \\
\hline GP15-9 & $\mathrm{sr}$ & - & + & per & w. & - & + & - \\
\hline GP15-24 & $\mathrm{sr}$ & - & + & per & w. & - & + & - \\
\hline GP15-25 & $\mathrm{sr}$ & - & + & per & w. trans. & - & + & - \\
\hline GP5-7 & sr & - & + & per & yell. & - & + & - \\
\hline W-3 & $\mathrm{r}$ & - & + & pol & br.w. & - & + & + \\
\hline G-8 & $\mathrm{r}$ & - & + & pol & yell. & - & + & + \\
\hline G-16 & $\mathrm{r}$ & - & + & pol & w. & - & + & + \\
\hline G-21 & sr & - & + & pol & br.w. & - & + & + \\
\hline GP15-15 & $r$ & - & + & pol & br.w. & - & + & + \\
\hline P-1 & $\mathrm{cr}$ & - & + & pol & w. trans. & - & + & + \\
\hline P-4 & $\mathrm{cr}$ & - & + & pol & w. & - & + & + \\
\hline P-5 & $\mathrm{cr}$ & - & + & pol & w. trans. & - & + & + \\
\hline P-8 & $\mathrm{cr}$ & - & + & pol & w. trans. & - & + & + \\
\hline P-15 & $\mathrm{cr}$ & - & + & pol & br.w. & - & + & + \\
\hline W-7 & $\mathrm{sr}$ & - & + & pol & br.w. & - & + & \pm \\
\hline GP5-20 & $\mathrm{r}$ & - & + & pol & yell. w. & - & + & + \\
\hline W-5 & sr & - & + & pol & br. w. & - & + & + \\
\hline W-12 & $\mathrm{sr}$ & - & + & pol & br. w. & - & + & + \\
\hline G-1 & $\mathrm{sr}$ & - & + & pol & br. w. & - & + & + \\
\hline G-2 & $\mathrm{sr}$ & - & + & pol & br.w. & - & + & + \\
\hline G-5 & $\mathrm{sr}$ & - & + & pol & yell. w. & - & + & + \\
\hline G-6 & $\mathrm{sr}$ & - & + & pol & w. trans. & - & + & + \\
\hline G-7 & $\mathrm{sr}$ & - & + & pol & br. w. & - & + & + \\
\hline G-9 & $\mathrm{sr}$ & - & + & pol & br.w. & - & + & + \\
\hline G-12 & $\mathrm{sr}$ & - & + & pol & br.w. & - & + & + \\
\hline G-13 & $\mathrm{sr}$ & - & + & pol & br.w. & - & + & + \\
\hline G-14 & $\mathrm{r}$ & - & + & pol & br. w. & - & + & + \\
\hline G-17 & $\mathrm{sr}$ & - & + & pol & br. w. & - & + & + \\
\hline G-19 & $\mathrm{sr}$ & - & + & pol & w. & - & + & + \\
\hline G-22 & $\mathrm{sr}$ & - & \pm & $?$ & br.w. & - & + & + \\
\hline G-24 & $\mathrm{sr}$ & - & + & pol & br. w. & - & + & + \\
\hline
\end{tabular}


characteristics of the strains.

\begin{tabular}{|c|c|c|c|c|c|c|c|c|c|c|c|c|c|c|}
\hline \multicolumn{6}{|c|}{ Hugh \& Leifson's test ${ }^{5)}$} & \multirow{3}{*}{$\begin{array}{l}\bar{O} \\
\bar{O}\end{array}$} & \multirow{3}{*}{$\begin{array}{l}\overrightarrow{\tilde{s}} \\
\frac{\hat{\alpha}}{\dot{u}} \\
\dot{\Sigma}\end{array}$} & \multirow{3}{*}{ 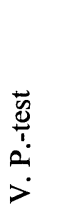 } & \multirow{3}{*}{ 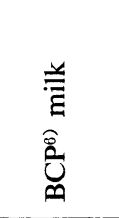 } & \multirow{3}{*}{ 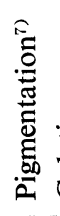 } & \multirow{3}{*}{ 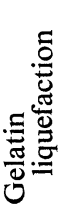 } & \multirow{3}{*}{$\begin{array}{l}.0 \\
.00 \\
0 \\
0 \\
0 \\
0 \\
\text { Z }\end{array}$} & \multirow{3}{*}{ 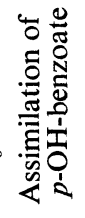 } & \multirow{3}{*}{ 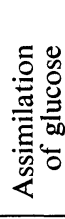 } \\
\hline \multicolumn{2}{|c|}{ gluc.* } & \multicolumn{2}{|c|}{ lact.** } & \multicolumn{2}{|c|}{ sucr. $* * *$} & & & & & & & & & \\
\hline open & close & open & close & open & close & & & & & & & & & \\
\hline $\mathrm{n}$ & $\mathrm{n}$ & al & $\mathrm{n}$ & ac & ac & - & - & - & & - & - & - & - & + \\
\hline $\mathrm{n}$ & $\mathrm{n}$ & al & $\mathrm{n}$ & al & $\mathrm{n}$ & - & - & -1 & red-pept & + & - & - & - & + \\
\hline al & $\mathrm{n}$ & al & $\mathrm{n}$ & al & $\mathrm{n}$ & - & - & - & al-coa & + & + & - & - & - \\
\hline al & $\mathrm{n}$ & ac & $\mathrm{n}$ & al & $\mathrm{n}$ & - & - & - & al-coa & + & + & - & - & - \\
\hline al & $\mathrm{n}$ & al & $\mathrm{n}$ & al & $\mathrm{n}$ & - & - & - & al-coa & + & + & - & - & - \\
\hline (al) & $\mathrm{n}$ & al & $\mathrm{n}$ & al & $\mathrm{n}$ & - & - & - & al-coa & + & + & - & - & - \\
\hline (al) & $\mathrm{n}$ & al & $\mathrm{n}$ & al & $\mathrm{n}$ & - & - & - & al-coa & + & + & - & - & - \\
\hline ac & (ac) & ac & $\mathrm{n}$ & al & $\mathrm{n}$ & - & - & $-a$ & ac-coa & + & - & - & + & \pm \\
\hline ac & (ac) & ac & $\mathrm{n}$ & al & $\mathrm{n}$ & - & - & - & ac-coa & + & - & - & + & - \\
\hline ac & (ac) & ac & $\mathrm{n}$ & al & $\mathrm{n}$ & - & - & - & ac-coa & + & - & - & + & - \\
\hline ac & (ac) & ac & $\mathrm{n}$ & al & $\mathrm{n}$ & - & - & - & ac-coa & + & - & - & + & - \\
\hline ac & ac & (al) & $\mathrm{n}$ & ac & ac & - & - & $-a$ & al-coa & + & + & + & - & \pm \\
\hline $\mathrm{ac}$ & $\mathrm{n}$ & (al) & $\mathrm{n}$ & $\mathrm{n}$ & ac & - & - & - & & - & - & - & - & - \\
\hline $\mathrm{n}$ & $\mathrm{n}$ & al & $\mathrm{n}$ & ac & ac & - & - & - & al-coa & - & - & - & - & + \\
\hline (ac) & $\mathrm{n}$ & al & ac & al & ac & - & - & -1 & red-pept & + & - & - & - & + \\
\hline (ac) & $\mathrm{n}$ & al & $\mathrm{n}$ & al & ac & - & - & $-a$ & & - & - & + & + & + \\
\hline (ac) & $\mathrm{n}$ & al & $\mathrm{n}$ & al & (ac) & - & - & $-a$ & & - & - & + & - & + \\
\hline $\mathrm{n}$ & $\mathrm{n}$ & (al) & $\mathrm{n}$ & (al) & $\mathrm{n}$ & - & - & $-\mathrm{a}$ & & - & - & - & - & - \\
\hline $\mathrm{n}$ & $\mathrm{n}$ & (al) & $\mathrm{n}$ & (al) & $\mathrm{n}$ & - & - & $-a$ & & - & - & - & - & - \\
\hline $\mathrm{n}$ & $\mathrm{n}$ & (al) & $\mathrm{n}$ & (al) & $\mathrm{n}$ & - & - & $-a$ & & - & - & - & - & - \\
\hline $\mathrm{n}$ & $\mathrm{n}$ & (al) & $\mathrm{n}$ & (al) & $\mathrm{n}$ & - & - & $-a$ & & + & - & - & - & - \\
\hline $\mathrm{n}$ & $\mathrm{n}$ & $\mathrm{n}$ & $\mathrm{n}$ & (al) & $\mathrm{n}$ & - & - & $-a$ & & - & - & + & - & - \\
\hline ac & $\mathrm{n}$ & al & $\mathrm{n}$ & (ac) & $\mathrm{n}$ & - & - & -1 & pept & + & + & - & \pm & + \\
\hline (ac) & $\mathrm{n}$ & $\mathrm{n}$ & $\mathrm{n}$ & (ac) & $\mathrm{n}$ & - & - & $-a$ & al-coa & + & + & + & + & + \\
\hline $\mathrm{ac}$ & $\mathrm{n}$ & al & $\mathrm{n}$ & ac & $\mathrm{n}$ & - & - & $-a$ & & + & - & + & \pm & + \\
\hline $\mathrm{ac}$ & $\mathrm{n}$ & al & $\mathrm{n}$ & (ac) & $\mathrm{n}$ & - & - & $-a$ & & + & - & + & \pm & \pm \\
\hline (ac) & $\mathrm{n}$ & al & $\mathrm{n}$ & ac & $\mathrm{n}$ & - & - & $-a$ & & - & - & + & \pm & + \\
\hline (ac) & $\mathrm{n}$ & al & $\mathrm{n}$ & al & $\mathrm{n}$ & - & - & $-a$ & & - & - & + & - & + \\
\hline (ac) & $\mathrm{n}$ & al & $\mathrm{n}$ & al & $\mathrm{n}$ & - & - & $-a$ & & - & - & + & + & + \\
\hline (ac) & $\mathrm{n}$ & al & $\mathrm{n}$ & (ac) & $\mathrm{n}$ & - & - & $-a$ & & - & - & + & + & + \\
\hline (ac) & $\mathrm{n}$ & al & $\mathrm{n}$ & al & $\mathrm{n}$ & - & - & $-a$ & & - & - & + & + & + \\
\hline (ac) & $\mathrm{n}$ & al & $\mathrm{n}$ & al & $\mathrm{n}$ & - & - & $-a$ & & - & - & + & + & + \\
\hline $\mathrm{ac}$ & $\mathrm{n}$ & al & $\mathrm{n}$ & al & $\mathrm{n}$ & - & - & $-a$ & & + & - & + & + & + \\
\hline $\mathrm{ac}$ & $\mathrm{n}$ & al & $\mathrm{n}$ & al & $\mathrm{n}$ & - & - & $-a$ & & + & - & + & + & + \\
\hline $\mathrm{ac}$ & $\mathrm{n}$ & al & $\mathrm{n}$ & al & $\mathrm{n}$ & - & - & $-a$ & & + & - & + & + & + \\
\hline (ac) & $\mathrm{n}$ & al & $\mathrm{n}$ & al & $\mathrm{n}$ & - & - & $-a$ & & - & - & + & + & + \\
\hline (ac) & $\mathrm{n}$ & al & $\mathrm{n}$ & al & $\mathrm{n}$ & - & - & $-a$ & & + & - & + & + & + \\
\hline (ac) & $\mathrm{n}$ & al & $\mathrm{n}$ & al & $\mathrm{n}$ & - & - & & & - & - & + & + & + \\
\hline (ac) & $\mathrm{n}$ & al & $\mathrm{n}$ & al & $\mathrm{n}$ & - & - & $-a$ & & - & - & + & + & + \\
\hline
\end{tabular}


Table 1.

\begin{tabular}{|c|c|c|c|c|c|c|c|c|}
\hline $\begin{array}{c}\text { Strain } \\
\text { No. }\end{array}$ & 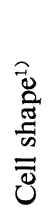 & 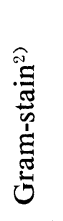 & 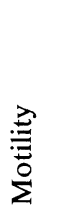 & 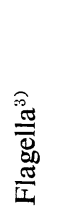 & 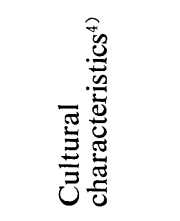 & 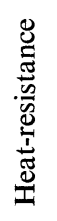 & $\frac{\ddot{\mathscr{Z}}}{\frac{\tilde{\pi}}{\pi}}$ & 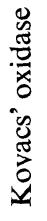 \\
\hline G-25 & $\mathrm{sr}$ & - & + & pol & br. w. trans. & - & + & + \\
\hline G-28 & sr & - & + & pol & br. w. & - & + & + \\
\hline GP15-30 & $\mathrm{sr}$ & - & + & pol & br. w. & - & + & + \\
\hline $\mathrm{W}-13$ & $\mathrm{r}$ & - & + & pol & br. w. & - & + & \pm \\
\hline$P-13$ & $\mathrm{cr}$ & - & + & $?$ & w. trans. & - & + & \pm \\
\hline W-8 & $\mathrm{r}$ & - & + & pol & br. w. & - & + & \pm \\
\hline W-11 & sr & - & + & pol & w. & - & + & + \\
\hline G-11 & $\mathrm{r}$ & - & + & pol & br. w. & - & + & + \\
\hline G-20 & $\mathrm{r}$ & - & + & pol & yell. w. & - & + & + \\
\hline $\mathrm{G}-23$ & $\mathrm{r}$ & - & + & pol & yell. w. & - & + & + \\
\hline GP5-6 & $\mathrm{r}$ & - & + & pol & br. w. trans. & - & + & + \\
\hline GP5-10 & $\mathrm{sr}$ & - & + & pol & br.w. & - & + & + \\
\hline GP5-15 & $\mathrm{r}$ & - & + & pol & br.w. & - & + & + \\
\hline GP5-16 & $\mathrm{r}$ & - & + & pol & w. trans. & - & + & + \\
\hline GP5-17 & $\mathrm{r}$ & - & + & pol & br. w. & - & + & + \\
\hline GP5-24 & $\mathrm{r}$ & - & + & pol & br. w. & - & + & + \\
\hline GP5-28 & $r$ & - & + & pol & br. w. & - & + & + \\
\hline G-10 & $\mathrm{r}$ & - & + & pol & yell. w. & - & + & + \\
\hline G-18 & $\mathrm{r}$ & - & + & pol & yell. & - & + & + \\
\hline G-26 & $\mathrm{r}$ & - & + & pol & yell. & - & + & + \\
\hline G-27 & $\mathrm{r}$ & - & + & pol & yell. w. & - & + & + \\
\hline$G-29$ & $\mathrm{r}$ & - & + & pol & yell. & - & + & + \\
\hline W-6 & lr & + & + & per & br. w. trans. & - & + & + \\
\hline GP5-4 & $\mathrm{r}$ & + & - & - & or. yell. & - & + & \pm \\
\hline GP15-3 & $\operatorname{lr}$ & + & - & - & or. w. & - & + & - \\
\hline GP15-5 & lr & + & + & $?$ & w. & - & + & - \\
\hline
\end{tabular}

1) sr, short rod; r, rod; cr, coccoidal rod; lr, long rod. ${ }^{2)}-$, gram-negative; + , grampositive. ${ }^{3)}$-, no flagella; pol, polar flagella; per, peritrichous flagella; N.D., not determined. 4) trans., translucent; br., brownish; w., whitish; or., orange; yell., yellowish. 5) open, aerobic; close, anaerobic; al, alkaline; ac, acidic; $n$, neutral; the letters in parentheses indicate weak reaction.

extract requirement. The following organic compounds were tested as sole sources of carbon and energy or sole sources of carbon, nitrogen, and energy: glutamate, glycine, leucine, lysine, phenylalanine, casamino acid, arabinose, fructose, galactose, glucose, inositol, maltose, mannitol, mannose, xylose, acetate, butyrate, citrate, fumarate glutarate maleate, malonate, propionate, succinate, and valerate. The basal salt solution was supplemented with $\mathrm{NH}_{4} \mathrm{NO}_{3}(0.2 \% \mathrm{wt} / \mathrm{vol})$ 
(Continued).

\begin{tabular}{|c|c|c|c|c|c|c|c|c|c|c|c|c|c|c|}
\hline \multicolumn{6}{|c|}{ Hugh \& Leifson's test ${ }^{5)}$} & \multirow{3}{*}{$\bar{D}$} & \multirow{3}{*}{$\begin{array}{l}\overrightarrow{\tilde{s}} \\
\frac{0}{1} \\
\ddot{u} \\
\dot{\Sigma}\end{array}$} & \multirow{3}{*}{ 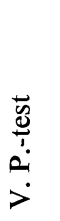 } & \multirow{3}{*}{ 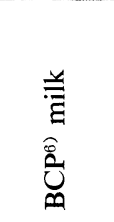 } & \multirow{3}{*}{ 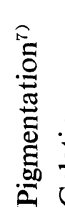 } & \multirow{3}{*}{ } & \multirow{3}{*}{ 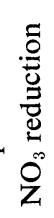 } & 范 & \multirow{2}{*}{ 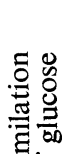 } \\
\hline \multicolumn{2}{|c|}{ gluc.* } & \multicolumn{2}{|c|}{ lact.** } & \multicolumn{2}{|c|}{ sucr.*** } & & & & & & & & 竞 & \\
\hline open & close & open & close & open & close & & & & & & & & & \\
\hline (ac) & $\mathrm{n}$ & al & $\mathrm{n}$ & al & $\mathrm{n}$ & - & - & - & & - & - & + & + & + \\
\hline (ac) & $\mathrm{n}$ & al & $\mathrm{n}$ & al & $\mathrm{n}$ & - & - & - & & - & - & + & + & + \\
\hline$(\mathrm{ac})$ & $\mathrm{n}$ & al & $\mathrm{n}$ & al & $\mathrm{n}$ & - & - & - & al-coa & + & - & + & + & - \\
\hline (ac) & $\mathrm{n}$ & $\mathrm{n}$ & $\mathrm{n}$ & (ac) & $\mathrm{n}$ & - & - & - & ac-coa & - & - & + & \pm & + \\
\hline ac & $\mathrm{n}$ & ac & $\mathrm{n}$ & al & $\mathrm{n}$ & - & - & - & ac-coa & + & - & + & - & + \\
\hline ac & $\mathrm{n}$ & al & $\mathrm{n}$ & ac & $\mathrm{n}$ & - & - & - & & - & - & - & - & + \\
\hline ac & $\mathrm{n}$ & al & $\mathrm{n}$ & (ac) & $\mathrm{n}$ & - & - & - & & - & - & - & - & + \\
\hline (ac) & $\mathrm{n}$ & al & $\mathrm{n}$ & $\mathrm{n}$ & $\mathrm{n}$ & - & - & - & al-coa & + & + & - & + & - \\
\hline (ac) & $\mathrm{n}$ & al & $\mathrm{n}$ & al & $\mathrm{n}$ & - & - & - & al-pept & + & - & - & + & + \\
\hline (ac) & $\mathrm{n}$ & al & $\mathrm{n}$ & al & $\mathrm{n}$ & - & - & - & al-coa & + & - & - & + & + \\
\hline (ac) & $\mathrm{n}$ & $\mathrm{n}$ & $\mathrm{n}$ & $\mathrm{n}$ & $\mathrm{n}$ & - & - & - & & - & - & - & + & + \\
\hline (ac) & $\mathrm{n}$ & $\mathrm{n}$ & $\mathrm{n}$ & al & $\mathrm{n}$ & - & - & - & & - & - & - & + & + \\
\hline (ac) & $\mathrm{n}$ & $\mathrm{n}$ & $\mathrm{n}$ & al & $\mathrm{n}$ & - & - & - & & - & - & - & + & + \\
\hline (ac) & $\mathrm{n}$ & $\mathrm{n}$ & $\mathrm{n}$ & al & $\mathrm{n}$ & - & - & - & & - & - & - & + & + \\
\hline (ac) & $\mathrm{n}$ & $\mathrm{n}$ & $\mathrm{n}$ & al & $\mathrm{n}$ & - & - & - & & - & - & - & + & + \\
\hline (ac) & $\mathrm{n}$ & $\mathrm{n}$ & $\mathrm{n}$ & al & $\mathrm{n}$ & - & - & - & & - & - & - & + & + \\
\hline (ac) & $\mathrm{n}$ & $\mathrm{n}$ & $\mathrm{n}$ & al & $\mathrm{n}$ & - & - & - & & - & - & - & + & + \\
\hline (ac) & $\mathrm{n}$ & al & $\mathrm{n}$ & ac & $\mathrm{n}$ & - & - & - & ac-coa & + & - & - & + & + \\
\hline (ac) & $\mathrm{n}$ & al & $\mathrm{n}$ & $\mathrm{n}$ & $\mathrm{n}$ & - & - & - & red-pept & + & - & - & - & + \\
\hline (ac) & $\mathrm{n}$ & al & $\mathrm{n}$ & al & $\mathrm{n}$ & - & - & - & red-pept & + & - & - & - & + \\
\hline (ac) & $\mathrm{n}$ & al & $\mathrm{n}$ & al & $\mathrm{n}$ & - & - & - & ac-coa & + & - & - & - & + \\
\hline (ac) & $\mathrm{n}$ & al & $\mathrm{n}$ & al & $\mathrm{n}$ & - & - & - & red-pept & + & - & - & - & + \\
\hline $\mathrm{n}$ & $\mathrm{n}$ & $\mathrm{ac}$ & ac & $\mathrm{ac}$ & ac & - & - & - & ac-coa & - & - & + & - & - \\
\hline $\mathrm{n}$ & $\mathrm{n}$ & $\mathrm{n}$ & $\mathrm{n}$ & $\mathrm{n}$ & $\mathrm{n}$ & - & - & - & ac-coa & + & + & - & - & + \\
\hline ac & (ac) & (ac) & $\mathrm{n}$ & (ac) & (ac) & - & - & - & al-coa & - & + & - & - & + \\
\hline ac & (ac) & al & $\mathrm{n}$ & (ac) & (ac) & - & - & - & al-coa & - & + & + & - & + \\
\hline
\end{tabular}

* glucose, ${ }^{* *}$ lactose, ${ }^{* * *}$ sucrose. ${ }^{\text {})}$ al, alkaline; ac, acidic; al-coa, coagulation of milk after alkaline reaction; ac-coa, coagulation of milk after acidic reaction; pept, peptonization; redpept, decoloration of BCP and peptonization; N.D., not determined. ${ }^{7)}$ water soluble greenish yellow pigment.

for the test of organic acids and saccharides.

All chemicals were purchased from Wako Pure Chemical Industries Ltd., Tokyo, except the yeast extract which came from Difco Laboratories, Detroit, Michigan, and the polypepton and the meat extract which came from Kyokuto Chemical Industries Ltd., Tokyo.

Electron microscopy. All of the strains were grown on albumin agar medium for $48 \mathrm{hr}$, and the cell specimens were shadowed with carbon and chromium through 
vacuum evaporation. Electron micrographs were obtained with a Nihon Denshi model JEM-T6S transmission electron microscope.

\section{RESULTS AND DISCUSSION}

\section{Bacteriological characteristics of the isolated strains}

Table 1 shows some of the bacteriological characteristics of the strains. Almost all of the strains were gram-negative and rod-shaped cells irrespective of their sources, and many of them were motile with polar flagella although some moved with peritrichous flagella. All of the strains were catalase positive and not heat-resistant. They were also negative in indol production, methyl red and VogesProskauer tests. Good correlation existed between the types of flagellation and the results of Kovacs' oxidase test; all the strains with peritrichous flagella were negative to the test and those with polar flagella were positive.

Their cultural characteristics on agar slant were diverse; they varied in pigmentation from white to brownish white, translucent white, yellowish white, yellow and orange, but yellow- or orange-pigmented strains were decidedly in the minority. Few strains had fermentative characteristics in acid production from glucose under Hugh and Leifson's test, and although a few of the strains showed no acid production from glucose oxidatively or fermentatively, most of them were oxidative in the test. Therefore, a majority of the strains may be included under the following characteristics; gram-negative, rod-shaped and polarly flagellated cells, Kovacs' oxidase positive and oxidative acid productive in Hugh and Leifson's test.

Reaction to $\mathrm{BCP}$ milk, gelatin liquefaction and nitrate reduction varied with the strains. This means that they are taxonomically diverse. There were four strains of gram-positive bacteria, W-6, GP5-4, GP15-3 and GP15-5, and two strains of non-motile, gram-negative and yellow- or orange-pigmented bacteria, G-3 and G-15.

\section{Grouping the strains and bacterial flora in the percolated soils}

In order to differentiate readily the bacterial strains into their respective groups, the present author developed a diagnostic key based on a combination of the bacteriological characteristics of the strains as indicated in SKERMAN's manual (19). Since a great majority of the strains were gram-negative, the differentiation was focussed on the gram-negative bacterial strains (Fig. 1). The gram-negative bacterial strains were first divided into seven groups, groups I, II, III, IV, V, VI and VII, based on the characteristics of motility, pigmentation, flagellation, results of Kovacs' oxidase test and reactions to Hugh and Leifson's test. All of the strains of group II liquefied gelatin but those of group III did not. Although these two groups, II and III were thought to be closely allied, they were assigned separately in view of a tentative taxonomic grouping of the isolates. 


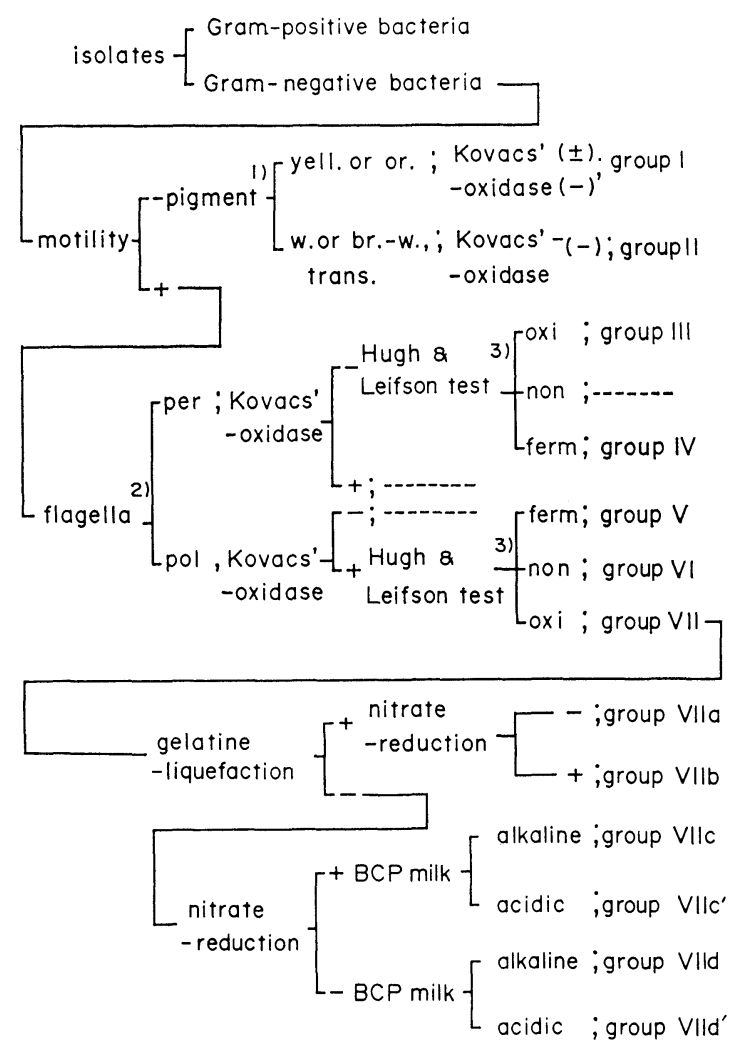

Fig. 1. A diagnostic scheme for grouping the strains.

Genera subsumed in each group $(21,22)$ : I, Not subsumed; II, Acinetobacter; III, not subsumed; IV, not subsumed; V, not subsumed; VI, Pseudomonas; VII, Pseudomonas groups other than that of group VI; VIIa, VIIb, VIIc, VIIc', VIId and VIId' denote different types of Pseudomonas of group VII.

Many of the strains fell into group VII, but they were diverse in the following characteristics; gelatin liquefaction, nitrate reduction and reaction to BCP milk. Therefore, group VII was further divided into six subgroups, groups VIIa, VIIb, VIIc, VIIc', VIId and VIId', based on a combination of the above three characteristics.

Sixty five strains of the tested bacteria may be classified as follows:

group I: G-3, G-15

group II: W-1, W-2, W-9, W-14, W-15

group III: GP15-1, GP15-9, GP15-24, GP15-25

group IV: GP5-7

group V: W-3, G-8, G-16, G-21, GP15-15

group VI: P-1, P-4, P-5, P-8, P-15 
group VIIa: W-7

group VIIb: GP5-20

group VIIc: W-5, W-12, G-1, G-2, G-5, G-6, G-7, G-9, G-12, G-13, G-14, G-17, G-19, G-22, G-24, G-25, G-28, GP15-30

group VIIc': W-13, P-13

group VIId: W-8, W-11, G-11, G-20, G-23, GP5-6, GP5-10, GP-5-15, GP516, GP5-17, GP5-24, GP5-28

group VIId': G-10, G-18, G-26, G-27, G-29

Gram-positive bacteria: W-6, GP5-4, GP15-3, GP15-5

Group III showed acidic reaction to glucose under anaerobic condition (close) in Hugh and Leifson's test, but the reaction, which appeared soon after incubation started, remained weak even after long incubation time. Therefore, the acidic reaction appeared to be brought about by incomplete exclusion of oxygen from the medium covered with liquid paraffin. For this the group was judged to be oxidative.

The type of flagellation of strain P-13, which was motile under the microscopic observation of the cells by the hanging-drop method, could not be confirmed. It was placed in group VII, however, because of its positive reaction to Kovacs' oxidase test. Although the strains G-3 and G-15 were included in the same group,

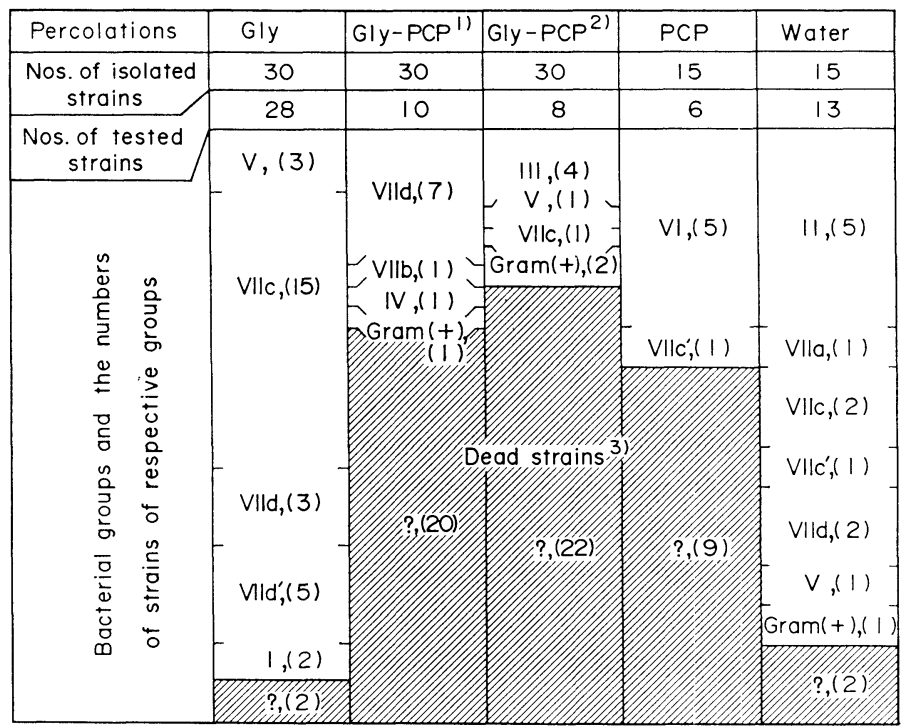

Fig. 2. A schematic depiction of soil bacterial flora and predominance of respective bacterial groups.

Number in parentheses after each group mark indicates the number of strains of the group in both live and dead strains ${ }^{1)}$ five days' percolation, ${ }^{2)}$ fifteen days' percolation, and ${ }^{3)}$ strains that died after purification. 
they were different in the OF-test; G-3 was fermentative but G-15 was not. Group III seemed to be closely akin to the genus Achromobacter or Agrobacterium, but the strains produced water soluble pigment. Therefore, it is difficult to determine the generic position of the group.

The bacterial floras vary among the different percolated soils (Fig. 2). Many strains isolated from the percolated soils other than the glycine- and water-percolated soils died within a half month after the purified cultures were stored. So they could not be bacteriologically characterized. Therefore, it is difficult to comment precisely on the difference of the bacterial floras in the respective percolated soils except for those in the glycine- and water-percolated soils. Bacterial flora differed markedly between glycine- and water-percolated soils; one group predominated in the former soil (group VIIc, over $50 \%$ ), while various groups were evenly isolated from the latter soil. A similar pattern of bacterial flora in the percolated soils was also observed in a previous study (24).

The surviving bacterial groups isolated from the percolated soils treated with PCP varied with the soils. They also differed from those in both glycine- and water-percolated soils without PCP treatment. Of the surviving groups isolated on the fifth day of percolation, group VIId predominated in the glycine-percolated soil with PCP treatment. However, the pattern of predominance of one group became distorted with the lapse of time; on the fifteenth day groups III, V, VIIc and the gram-positive bacteria were evenly distributed in the flora. In the PCP-percolated soil without glycine group VI predominated. This means that PCP modifies the bacterial community in soil and this modification is affected by soil conditions such as the nutrients available for microorganisms. In comparing the flora in soils percolated with and without PCP it appears that PCP allows only a few specific groups of bacteria to grow by restricting the proliferation of various other groups.

There is another possible explanation for the modification of bacterial flora by PCP in view of the dead strains, almost all of which were isolated from the PCP-treated soils. Over $60 \%$ of the isolated strains in PCP-treated soils died. If the dead strains all fall into the same bacterial group, PCP may be considered to establish a limited bacterial group in the soil irrespective of the difference in the nutritional condition of the soil.

In any event it is surprising that so many bacterial strains died within a half month of storage. Sometimes we unintentionally fail to maintain viable stock cultures because of improper management. In the present study, however, it is not likely that all of the dead strains were the result of mismanagement, since many strains from the PCP-percolated soil died while only a few from non-PCP-percolated soils died. So it appears that in the percolated soils, PCP may allow the proliferation of some limited groups of bacteria which are liable to die under the condition of laboratory culture.

PCP did not affect either glycine degradation or population change in the heterotrophic bacteria in the glycine-percolated soil (15). Since the increased 
heterotrophic bacteria participated mainly in degrading glycine in the percolated soil (25), PCP appears to effect no change in the bacterial flora, judging from the pattern of glycine degradation and the accompanying change in the population of the bacteria. However, a different bacterial flora was established with PCP treatment of glycine-percolated soil than without it. Therefore, it is important to determine the change in the microbial community to know the effect of pesticides on microorganisms in soil.

While the number of bacteria decreased rapidly after reaching the maximum level in the glycine-percolated soil without PCP, the population in the glycinepercolated soil with PCP attained and sustained maximum numbers for some time (15). Therefore, the bacterial flora in the latter soil apparently is not altered during some period of percolation. However, the bacterial community of the persistent population was partly modified during percolation from the 5 th day to the 15th day, admitting that all of the dead GP5- and GP15-strains would be classified in the same group. In view of the alteration of floral composition in a stationary population, it is important to study the changes in both floral composition and population numbers when assessing the effects of pesticides in soil.

\section{Nutritional aspects of the isolates}

The P-strains used only a few kinds of organic compounds compared with the W-strains, GP5-strains, GP15-strains, and G-strains: All of the P-strains grew on casamino acid plus yeast extract and succinate as the other strains did, but many of them did not grow on other organic compounds most of which supported the other strains (Table 2). This suggests that the P-strains have more complex nutritional requirements than the other strains and this correlates the difference in taxonomic position of these strains in relation to the others.

Of the amino acids tested, phenylalanine was the one used least by the strains isolated from the glycine-percolated soils, irrespective of the addition of PCP. It was followed by leucine and lysine which were also little used. All of the strains used glutamate. This pattern of amino acid use was similarly reported in a previous work (26).

Glycine utilization by the GP5- and GP15-strains was not so great as by the G-strains. Most of the G-strains used glycine, but some did not, and the growth of the glycine-utilizing strains was poor. Glycine degradation and population increase in the glycine-percolated soils went on similarly whether the soils were treated with PCP or not (15). The proliferating heterotrophic bacteria in the glycine-percolated soils were, therefore, apparently participating in degrading the glycine. The unexpected impotence of glycine-utilization by the GP5- and GP15strains suggests that other strains, which may include the dead bacterial group(s), are likely to take part in glycine degradation in the glycine-percolated soil with PCP treatment. This also implies that, even though the process of a chemical reaction carried out by microorganisms and the population increase of micro- 
Table 2. Assimilation of organic compounds by the strains.

\begin{tabular}{|c|c|c|c|c|c|}
\hline \multirow{3}{*}{ Substrates tested } & \multicolumn{5}{|c|}{ Numbers of tested strains } \\
\hline & W-strains & P-strains & GP5-strains & GP15-strains & G-strains \\
\hline & 13 & 6 & 10 & 8 & 28 \\
\hline Glutamate & $11^{*}$ & 1 & 10 & 8 & 27 \\
\hline Glycine & $3(2)$ & 0 & $6(5)$ & $5(5)$ & 26 \\
\hline Leucine & 8 & 0 & $6(5)$ & 6 & $27(2)$ \\
\hline Lysine & $6(5)$ & 0 & $3(2)$ & 1 & 16 \\
\hline Phenylalanine & $7(1)$ & 0 & $2(1)$ & $7(3)$ & 24 (19) \\
\hline Casamino acid & 12 & 2 & 10 & 8 & 28 \\
\hline \multicolumn{6}{|l|}{ Casamino acid } \\
\hline+ yeast extract & 13 & 6 & 10 & 8 & 28 \\
\hline Arabinose & 4 & 0 & $8(1)$ & 4 & $13(6)$ \\
\hline Fructose & $4(1)$ & $1(1)$ & $9(1)$ & 6 & $20(17)$ \\
\hline Galactose & $7(3)$ & $2(2)$ & 9 & $7(1)$ & $28(17)$ \\
\hline Glucose & $6(1)$ & 1 & $10(1)$ & $4(0)$ & 27 \\
\hline Inositol & $5(3)$ & $1(1)$ & $3(2)$ & 6 & $19(14)$ \\
\hline Maltose & $6(3)$ & 0 & $8(6)$ & 7 & $21(18)$ \\
\hline Mannitol & $7(1)$ & $1(1)$ & $10(4)$ & $7(1)$ & $28(13)$ \\
\hline Mannose & $7(1)$ & $1(1)$ & $9(2)$ & 6 & $26(25)$ \\
\hline Xylose & $11(4)$ & $1(1)$ & $9(3)$ & 5 & $25(19)$ \\
\hline Acetate & 0 & 0 & $5(3)$ & $7(1)$ & $26(1)$ \\
\hline Butyrate & 0 & 0 & $8(2)$ & $7(1)$ & $25(2)$ \\
\hline Citrate & 1 & 1 & $9(1)$ & 6 & $25(4)$ \\
\hline Fumarate & $4(1)$ & 1 & $10(7)$ & 7 & $26(2)$ \\
\hline Glutarate & $10(2)$ & 0 & $8(7)$ & $6(1)$ & $26(1)$ \\
\hline Maleate & $5(5)$ & 0 & $8(1)$ & $6(4)$ & $12(6)$ \\
\hline Malonate & 2 & 0 & 9 & 7 & 18 \\
\hline Propionate & $7(1)$ & 0 & 8 & 7 & $20(2)$ \\
\hline Succinate & $11(2)$ & $6(1)$ & $10(1)$ & 7 & 27 \\
\hline Valerate & 10 & 0 & $9(1)$ & 6 & 17 \\
\hline
\end{tabular}

* Numbers in each column indicate those of growing strains, and numbers in parentheses, those of poorly growing strains of the growing ones.

organisms appear similarly among the plots of the experiment, the extent to which the particular bacterial groups responsible for the reaction are different must be considered. Again the importance of elucidation of bacterial flora together with their physiological or nutritional characteristics should be emphasized in the study of the effect of pesticides on bacteria in soil.

Further studies of the modification of bacterial flora in connection with their tolerance to PCP will be reported elsewhere.

The present study was supported by the Grants-in-Aid for Scientific Research from the Ministry ofEducation, Science and Culture of Japan (B8-R12-8 and B8-R12-9). The author expresses his gratitude to Emeritus Prof. C. Furusaka for his incessant encouragement during the work. 


\section{REFERENCES}

1) L. J. Audus, Plant Soil, 2, 31 (1949).

2) I. Watanabe and S. Hayashi, J. Soil Sci. Manure, Jpn., 43, 119 (1972).

3) M. A. Loos, R. N. Roberts, and M. Alexander, Can. J. Microbiol., 13, 679 (1967).

4) I. Watanabe, Soil Sci. Plant Nutr., 19, 109 (1973).

5) D. A. van Schreven, D. J. Lindenbergh, and A. Koridon, Plant Soil, 33, 513 (1970).

6) N. Balicka and J. Sobieszczanski, Acta Microbiol. Pol., 1, 7 (1969).

7) A. Debona and L. J. Audus, Weed Res., 10, 250 (1970).

8) C. M. Tu, Can. J. Plant Sci., 53, 401 (1973).

9) J. P. Voets, P. Meerschman, and W. Verstraete, Soil Biol. Biochem., 6, 149 (1974).

10) H. B. Gunner, B. M. Zuckerman, R. W. Walker, C. W. Miller, K. H. Deubert, and R. E. Longley, Plant Soil, 25, 249 (1966).

11) T. Matsuguchi and S. Ishizawa, J. Soil Sci. Manure, Jpn., 39, 241 (1968).

12) L. D. Houseworth and B. G. Tweedy, Plant Soil, 38, 493 (1973).

13) K. Nauman, Zbl. Bakt. Abt., II., 127, 379 (1972).

14) H. Kato, K. Sato, and C. Furusaka, Bull. Inst. Agric. Res. Tohoku Univ., 32, 1 (1980).

15) K. Sato, Plant Soil, 75, 417 (1983).

16) Tokyo Daigaku Densenbyo Kenkyujo Gakuyukai (ed.), Saikingaku Jisshu Teiyo, Maruzen, Tokyo (1958), p. 123.

17) W. F. Harrigan and M. E. McCance, Laboratory Methods in Microbiology, Academic Press, London and New York (1966), p. 207.

18) N. Kovacs, Nature (London), 178, 703 (1956).

19) V.B.D. SKerman, A Guide to the Identification of the Genera of Bacteria, 2nd ed., Williams \& Wilkins Co., Baltimore (1967).

20) W. F. Harrigan and M. E. McCance, Laboratory Methods in Microbiology, Academic Press, London and New York (1966), p. 194.

21) Bergey's Manual of Determinative Bacteriology, 8th ed., ed. by R. E. Buchanan and N. E. GibBons, Williams \& Wilkins Co., Baltimore (1974), p. 217, 290.

22) Bergey's Manual of Systematic Bacteriology, Vol. 1, ed. by N. R. KRIEG, Williams \& Wilkins Co., London (1984), p. 140.

23) N. Kovacs, $Z$. Immunforsch. Exp. Ther., 55, 311 (1928).

24) K. Sato, Bull. Inst. Agric. Res. Tohoku Univ., 22, 93 (1971).

25) C. Furusaka and K. Sato, Can. J. Biochem., 43, 1017 (1965).

26) K. Sato and C. FurusaKa, Trans. 10th Int. Congress Soil Sci. (Moscow), III, 108 (1974). 